\title{
Anerkennung Praxis-OP / OP I in der Praxis des freipraktizierenden Arztes
}

Tarifdienst FMH

Im Zusammenhang mit der Dignitätserhebung der FMH und den damit verbundenen wohlerworbenen Rechten sind Fragen betreffend Übergangsfristen bei den Praxis-OPs aufgetaucht. Der Artikel soll darlegen, wie das Anerkennungsverfahren abläuft und welche Fristen zu beachten sind.

\section{Definition Praxis-OP / OP I}

Wie viele Anfragen zeigen, ist die Definition des Praxis-OPs nicht eindeutig. In der Praxis des Grundversorgers wird der Eingriffsraum meist als Praxis-OP bezeichnet. Für diesen Raum gilt aber nach TARMED die Sparte Untersuchungsund Behandlungsraum (UBR). Der Praxis-OP nach TARMED ist aber Raum, welcher einem Operationssaal einer Klinik nahezu entspricht. So kann dieser Raum nicht als zusätzliches Sprechzimmer genutzt werden, wie dies in den meisten Grundversorgerpraxen der Fall ist.

Der OP I hingegen entspricht einem Normoperationssaal eines Spitals. Dieser Saal ist massiv grösser, als der Praxis-OP. Viele vor allem chirurgische Praxen verfügen aber über Säle, welche die Anforderungen eines OP I erfüllen.

\section{Abrechnung nach TARMED}

Für die Abrechnung der Leistungen sind verschiedene Positionen geschaffen worden. Primär können alle TARMED-Leistungen, die mit Sparte OP I tarifiert sind, auch in einem Praxis-OP durchgeführt werden. Für die Abrechnung des Praxis-OP kann erstens pro Sitzung die Grundtaxe 35.0010 abgerechnet werden. Bei allen in dieser Sitzung erbrachten Leistungen muss aber die TL-Komponente um 40\% abgesenkt werden (gemäss Position 35.0020).

Werden die Leistungen in einem anerkannten OP I durchgeführt, so kann pro Sitzung eine technische Grundleistung OP I (35.0030) abgerechnet werden. Die TL-Komponenten der einzelnen Leistungen können ohne Abzug verrechnet werden.

\section{Anerkennung Praxis-OP / Übergangs- bestimmungen}

Bereits der alte, blaue SUVA-Tarif kannte einen Sondertarif für Leistungen, welche im anerkannten Praxis-OP durchgeführt wurden. Diese Leistungen wurden mit einem Zuschlag von 80\% anstelle 30\% entschädigt. Unter TARMED können diese Leistungen nur noch abgerechnet werden, wenn ein anerkannter Praxis-OP vorhanden ist. Alle Praxen, welche über keine Anerkennung für diese Sparte verfügen, können die Leistungen, welche in TARMED mit Sparte OP I tarifiert sind, nicht mehr abrechnen.

Wie zu erwarten ist, führt diese Regelung nun bei nicht wenigen Praxen zu Problemen, da ein grosser Teil des Leistungsspektrums nicht mehr abgerechnet werden kann. Eine Anerkennung ist aber nicht möglich, da einige der TARMEDVorgaben nicht erfüllt werden. Die FMH hat nun mit den Kostenträgern vereinbart, dass während einer Übergangsfrist von einem Jahr auch PraxisOPs anerkannt werden können, welche die Vorgaben nicht komplett erfüllen. Das Verfahren für die Anerkennung bleibt auch für diese Praxen gleich. Die provisorisch anerkannten Praxen erhalten eine Liste mit den nicht erfüllten Punkten. Wer innerhalb eines Jahres die aufgelisteten Mängel behebt, erhält die definitive Anerkennung. Alle anderen können ab diesem Zeitpunkt keine Praxis-OP-Leistungen mehr abrechnen. Ausnahmen bestehen in den Kantonen, die bereits eigene Anerkennungen für Praxis-OPs im KVG-Bereich kennen (gemäss Absprachen zwischen Krankenversicherern und kantonalen Ärztegesellschaften). Diese Praxis-OPs behalten während dreier Jahre nach Einführung des TARMED die Anerkennungen im KVG-Bereich. Nach Ablauf von drei Jahren müssen aber auch diese Praxis-OPs die TARMED-Anforderungen voll erfüllen.

Verbunden mit der Anerkennung ist ein sogenanntes Qualitätssicherungsprogramm. Jährlich wird ein Sample von 5\% der anerkannten Sparten durch einen Hygienefachmann überprüft. Die Kosten für diese Überprüfung werden auf alle Praxisinhaber aufgeteilt. 


\section{Anerkennung OP I in Praxen}

Entgegen vieler Meinungen ist es durchaus möglich, einen OP I in der Praxis zu betreiben. Wer die notwendigen Vorgaben erfüllt, erhält die Bewilligung, mittels der Tarifpositionen für OP I abzurechnen. Das Anerkennungsverfahren läuft analog dem Verfahren Praxis-OP über die Arbeitsgruppe Ambulante Chirurgie der PAKODIG / TARMED Suisse (Sekretariat c/o Tarifdienst FMH, fmhtarif@hin.ch ). Die Arbeitsgruppe triagiert die eingehenden Gesuche. Alle Praxis-OPs werden in der Spartendatenbank der FMH und alle OP I in Praxen werden in der Spartendatenbank von $\mathrm{H}+$ geführt. Freipraktizierende Ärzte können sich für eine Anerkennung nur an die FMH wenden. Eine Anmeldung via das H+-Programm zur Anerkennung der Norm-OPs in Spitälern ist nicht zulässig.

Wer über einen anerkannten OP I in der Praxis verfügt, bleibt aber im Qualitätskontrollprogramm der Praxis-OPs integriert.

\section{Vorgaben für Praxis-OP und OP I}

Die Anforderungen an die Sparten können dem Konzept zur Anerkennung von Sparten TARMED entnommen werden. Das Konzept ist auf dem Internet unter www.fmh.ch (Tarife $\rightarrow$ Anerken- nung Sparten [TARMED] $\rightarrow$ Konzept über die Anerkennung von Sparten) aufgeschaltet. Bei Unklarheiten bezüglich einzelner Anforderungen gibt der Tarifdienst der FMH gerne Auskunft.

\section{Rekursweg}

Gegen negative Entscheide der Arbeitsgruppe Ambulante Chirurgie kann innert 30 Tagen schriftlich bei der PAKODIG Rekurs eingelegt werden.

\section{Kosten der Anerkennung}

Die Kosten für die Anerkennung betragen Fr. 500.-. Durch diesen Betrag werden die Aufwände der Vertragsparteien für die Überprüfung und die Kosten des Sekretariates gedeckt. Die Kosten für einen Rekurs gegen den Entscheid der Arbeitsgruppe Ambulante Chirurgie betragen Fr. 300.-, zuzüglich die allfälligen Kosten für einen Augenschein vor Ort. Bei einem positiven Rekursentscheid werden die Kosten zurückerstattet.

Die Kosten für die jährlichen Qualitätskontrollen liegen bei etwa Fr. 200.-. Diese Kosten sollen aber nach Möglichkeit noch sinken.

Tabelle 1

Übersicht über die Anerkennungsbestimmungen für Praxis-OP, OP I und UBR.

\begin{tabular}{|c|c|c|c|}
\hline & Praxis-OP & OP I & $\begin{array}{l}\text { Untersuchungs- und } \\
\text { Behandlungsraum (UBR) }\end{array}$ \\
\hline Definition & $\begin{array}{l}\text { Entspricht nahezu einem } \\
\text { Operationssaal einer Klinik. } \\
\text { Kein Sprechzimmer }\end{array}$ & $\begin{array}{l}\text { Normoperationssaal } \\
\text { eines Spitals, massiv } \\
\text { grösser als Praxis-OP }\end{array}$ & $\begin{array}{l}\text { Eingriffsraum in der } \\
\text { Praxis des Grundversorgers }\end{array}$ \\
\hline $\begin{array}{l}\text { Abrechnung } \\
\text { nach TARMED }\end{array}$ & $\begin{array}{l}\text { Grundtaxe } 35.0010 \\
\text { pro Sitzung, aber für jede } \\
\text { erbrachte Leistung muss } \\
\text { die TL-Komponente um } \\
40 \% \text { abgesenkt werden } \\
\text { (gemäss Position 35.0020). }\end{array}$ & $\begin{array}{l}\text { Technische Grundleistung } \\
\text { OP I ( } 35.0030) \text { pro Sitzung. } \\
\text { Die TL-Komponenten } \\
\text { der einzelnen Leistungen } \\
\text { können ohne Abzug } \\
\text { verrechnet werden. }\end{array}$ & $\begin{array}{l}\text { gemäss entsprechender } \\
\text { Leistungsposition }\end{array}$ \\
\hline Anerkennung & \multicolumn{2}{|c|}{$\begin{array}{l}\text { notwendig, um Leistungen, die nach Sparte OP I } \\
\text { tarifiert sind, abzurechnen. Keine wohlerworbenen Rechte } \\
\text { auf Niveau der Sparten }\end{array}$} & nicht notwendig \\
\hline $\begin{array}{l}\text { Übergangs- } \\
\text { bestimmungen }\end{array}$ & \multicolumn{2}{|l|}{ Übergangsfrist von einem Jahr } & - \\
\hline Ausnahmen & \multicolumn{2}{|c|}{$\begin{array}{l}\text { in bestimmten Kantonen gemäss Absprachen zwischen } \\
\text { Krankenversicherern und kantonalen Ärztegesellschaften }\end{array}$} & - \\
\hline
\end{tabular}

